\title{
Acute Cervical Motor Radiculopathy Induced by Neck and Limb Immobilization in a Patient with Parkinson Disease
}

\author{
Toshio Shimizu, Tetsuo Komori and Hideaki Hayashi
}

\begin{abstract}
A 68-year-old woman with Parkinson disease (PD) presented with acute monoplegia of her left upper extremity after the neck and limb immobilization for several hours. Her sensory function was normal, and the chest X-ray showed left phrenic nerve palsy. Electrophysiological studies showed multi-segment muscle involvement ( $\mathrm{C} 3$ to $\mathrm{T} 1$ ) including denervation potentials and reduced interference of motor units in needle electromyography. $\mathrm{M}$ wave amplitude in peripheral nerve stimulation was preserved except for the ulnar nerve, suggesting both axonal injury and conduction block at the anterior spinal roots. The patient showed fair recovery in several months, suggesting sufficient reinnervation and recovery of conduction block. Incomplete root avulsion was thought to be the pathomechanism of acute cervical motor radiculopathy.
\end{abstract}

Key words: motor radiculopathy, phrenic nerve palsy, neck immobilization, Parkinson disease

(DOI: 10.2169/internalmedicine.45.1670)

\section{Introduction}

Akinesia is the main clinical feature of Parkinson disease (PD), and PD patients often encounter akinesia-associated secondary disorders, such as radial nerve palsy, focal rhabdomyolysis and skin decubitus ulcer (1-3). These complications come from long-sustained focal pressure, leading to localized injury of the peripheral nerve, muscle and skin as a result of inability to move the body or extremities for a long time. Body immobilization may also induce nonphysiological focal tensile stress or stretch of nerves. We report herein a PD patient with acute cervical motor radiculopathy and phrenic nerve palsy which developed after neck immobilization due to akinesia during a down-phase of PD. The patient exhibited unilateral palsy of the upper extremity and diaphragm, followed by fair recovery, and her sensory function was well preserved. We discuss the pathomechanism focusing on anatomical and physiological differences between anterior and posterior roots of the spinal cord.

\section{Case Report}

A 68-year-old woman was admitted to our hospital in
June 2003 because of muscle weakness of her left upper extremity. She had suffered from PD. Briefly summarizing her history of PD, she noticed gait disturbance and motion slowness at age 60 . At age 62 , postural instability and falling tendency developed, and her posture became stooped. Levodopa and dopamine agonist were effective. Since age 65, a wearing-off phenomenon had appeared on medication of carbidopa/levodopa (10/100 mg three times per day) and cabergoline ( $2 \mathrm{mg}$ once a day), and then she had not been able to walk without support during a down-phase at night. Since May 2003, her symptoms worsened, and one night she could not voluntarily move at all for more than 10 hours, maintaining a posture holding a basket by her neck and left arm. The next morning she was found by her daughter, and then she noticed muscle weakness of her left shoulder and upper extremity. She was admitted to our hospital after 7 days. On admission, the patient was alert and non-demented. She showed parkinsonism such as masked face, monotonous speech, mild rigidity of her right upper and bilateral lower limbs, bradykinesia, and postural instability. The Hoen \& Yahr stage of PD was three. In addition, she showed proximal-dominant muscle weakness of her left shoulder and upper extremity. The muscle weakness was including the left trapezius and deltoid muscles, both of which

Department of Neurology, Tokyo Metropolitan Neurological Hospital, Fuchu, Tokyo

Received for publication November 28, 2005; Accepted for publication April 14, 2006

Correspondence to Toshio Shimizu, Department of Neurology, Tokyo Metropolitan Neurological Hospital, 2-6-1 Musashidai, Fuchu, Tokyo 1830042 

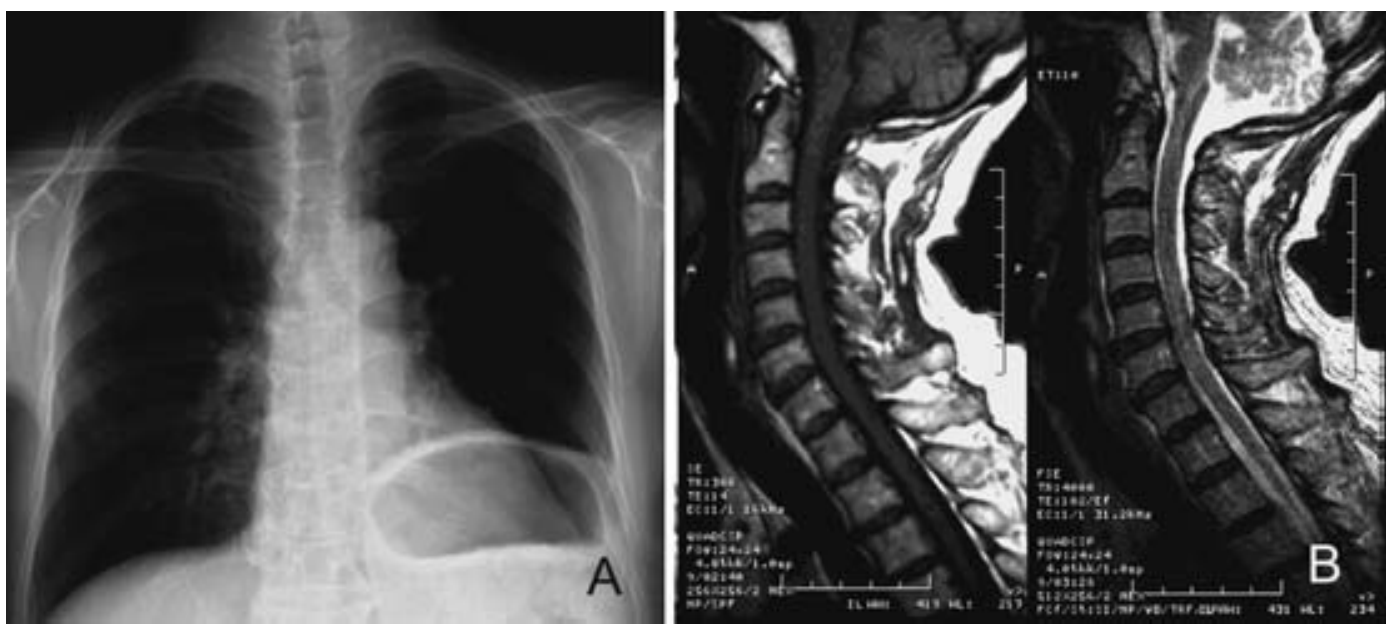

Figure 1. The chest X-ray film (A) and magnetic resonance images of cervical spine (B) on admission.
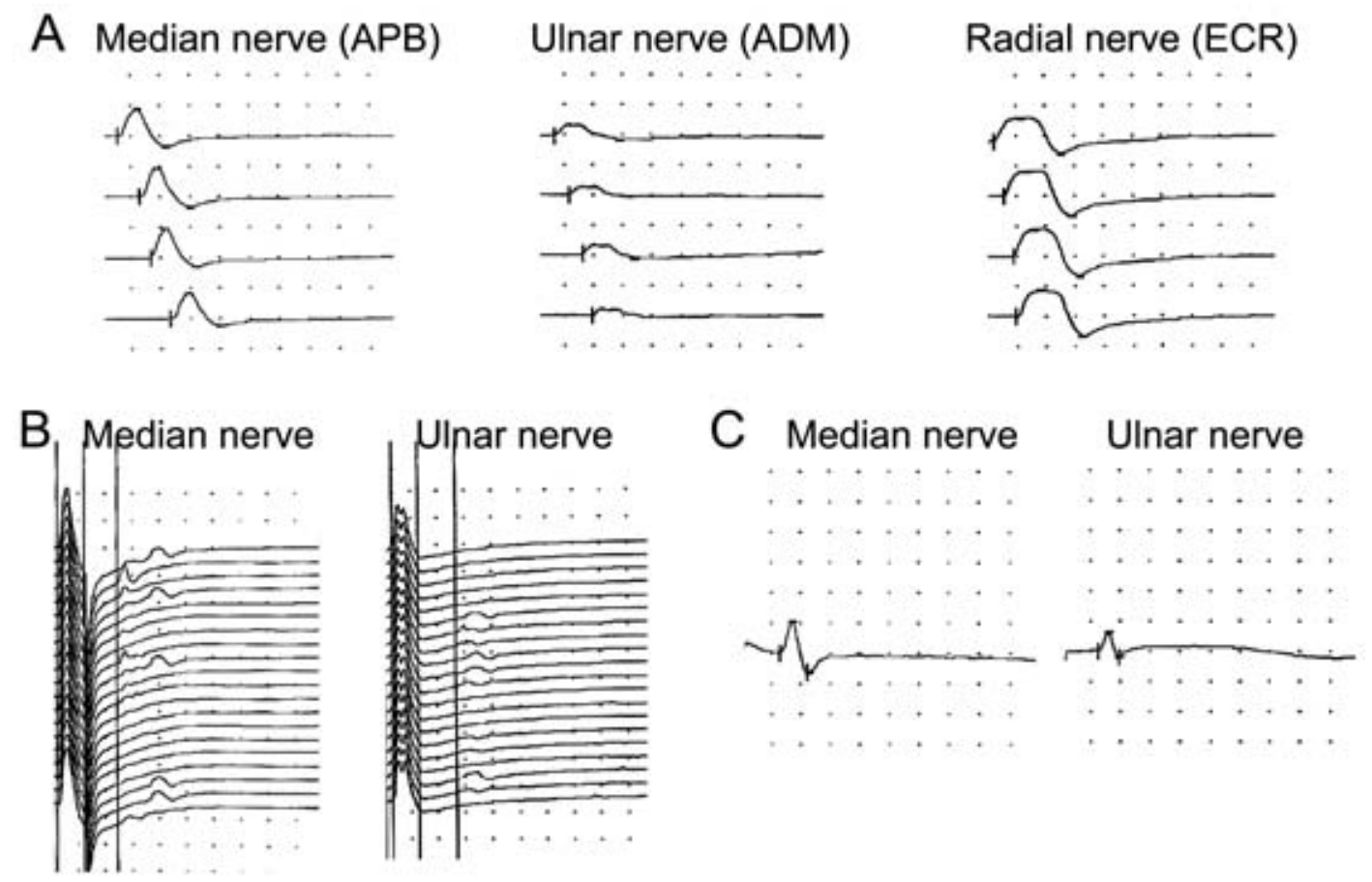

Figure 2. Nerve conduction study findings on admission. A: M waves of the left median, ulnar and radial nerves. More upper traces show the results of more distal electrical stimulation, and more lower traces those of more proximal electrical stimulation $(5 \mathrm{~ms} /$ division; $5 \mathrm{mV} /$ division in the median and radial nerve stimulation, $2 \mathrm{mV} /$ division in the ulnar nerve stimulation). B: $\mathrm{F}$ waves of the left median and ulnar nerves $(10 \mathrm{~ms} /$ division; $0.5 \mathrm{mV} /$ division $)$. C: Sensory nerve action potentials of the median and ulnar nerves ( $2 \mathrm{~ms} /$ division, $20 \mu \mathrm{V} /$ division). ADM, APB and ECR represent abductor digiti minimi, abductor pollicis brevis, and extensor carpi radialis muscles, respectively.

were most affected (level 1 by the manual muscle test), but the distal hand muscles were also weak (level 3). The major pectoralis muscle was intact. Her respiration was apparently normal. There was no weakness in the right upper and bilateral lower limbs. Deep tendon reflexes were diminished in the left upper extremity, and normal in the other limbs. The superficial and deep sensory function was normal in all extremities. There was no ataxia. After admission, she could walk with support during the up-phase of PD.

Normal laboratory studies included blood cell count, urinalysis, routine blood chemistry, hemoglobin-A1c, clotting and coagulating studies and CSF. Anti-nuclear, anti-DNA and anti-cardiolipin antibodies were negative. Her chest Xray film showed unilateral elevation of the left diaphragm (Fig. 1A). Pulmonary function test showed diminished vital capacity $(60.3 \%)$. Arterial oxygen and bicarbonate pressure 
A

Trapezius

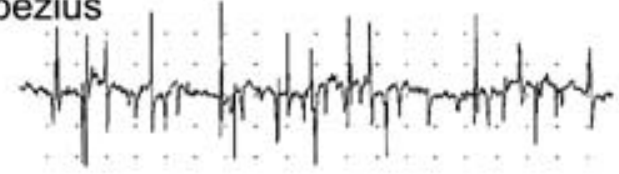

Biceps brachii
B
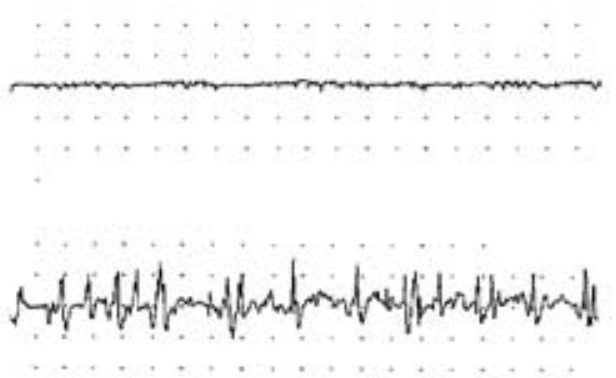

\section{Extensor carpi radialis}
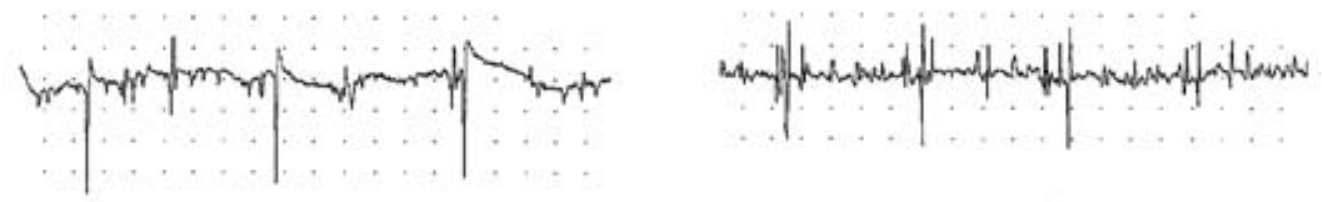

First dorsal interosseous
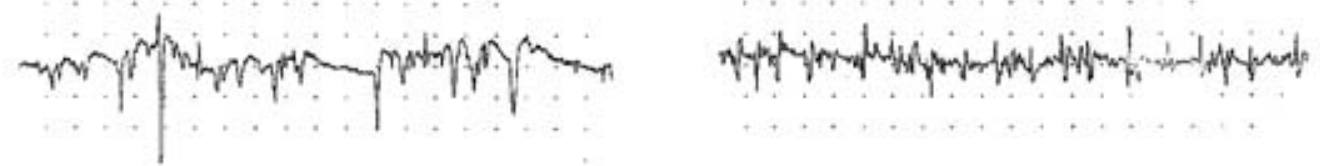

Figure 3. Needle EMG findings of the left trapezius, biceps brachii and first dorsal interosseous muscles. A: at rest $(20 \mathrm{~ms} /$ division; $50 \mu \mathrm{V} /$ division). B: during maximal muscle contraction (20 $\mathrm{ms} /$ division; 1mV/division).

were $67.9 \mathrm{mmHg}$ and $45.4 \mathrm{mmHg}$ in room air, respectively. Brain MR images showed old small cerebral infarction in the left (ipsilateral to the affected limb) basal ganglia. Cervical cord MR images showed localized intervertebral disk bulging at the level of $\mathrm{C} 5 / 6$, but the cord compression was very mild (Fig. 1B). The spinal canal was mildly stenotic, but there were no T2-weighted high intensity signals within the cervical cord.

For electrophysiological study, a peripheral nerve conduction study showed a reduced amplitude of $\mathrm{M}$ wave of the left ulnar nerve (abductor digiti minimi muscle) when compared with the intact side (Fig. 2A). There was no definite laterality in the $\mathrm{M}$ wave amplitude of the median nerve (abductor pollicis brevis) and radial nerve (extensor carpi radialis; ECR) (Fig. 2A). The $\mathrm{F}$ wave frequency of the left median and ulnar nerve was reduced (Fig. 2B). Motor nerve conduction velocity was within normal range in the examined nerves. Sensory nerve conduction velocity and action potentials of the left median and ulnar nerves were also intact (Fig. 2C). Needle electromyography (EMG) was examined on admission for the bilateral trapezius, biceps brachii, ECR and first dorsal interosseous (FDI) muscles. The left trapezius, ECR and FDI showed denervation potentials (positive sharp wave and fibrillation potential) at rest (Fig. 3A). During maximal voluntary muscle contraction, all the muscles of the left side showed reduced interference pattern, whereas the right side muscle showed full interference (Fig. 3B). There was no high amplitude or long duration motor unit potentials at the first examination. M wave amplitude of the phrenic nerves obtained by the supramaximal electrical stimulation at the posterior edge of the sternocleidomastoid muscle were $0.34 \mathrm{mV}$ in the left and $0.50 \mathrm{mV}$ in the right, both of which were within normal range (normal $>0.2 \mathrm{mV}$; Fig. 4A) (4). Diaphragm needle EMG at the 8th intercostal level showed reduced interference of the motor units in the left side and full interference in the right side during the deep inspiratory phase (Fig. 4B) (5). No active denervation potentials were obtained from the diaphragm muscles.

Somatosensory evoked potentials to median nerve stimulation were bilaterally normal, that is, spinal N13s and parietal N20s were well evoked symmetrically. Central sensory conduction times (latency difference between N13 and N20) were also bilaterally normal. Transcranial magnetic stimulation over the hand motor area induced motor evoked potentials of smaller amplitude from the left FDI than from the right FDI. Central motor conduction times were normal for both muscles.

After admission, the weakness gradually began to improve spontaneously. One month after the onset, the left ulnar nerve stimulation showed an increase in $\mathrm{M}$ amplitude, and $\mathrm{F}$ wave frequency improved with no latency reduction. The motor unit potentials of each muscle in the needle EMG examination were increased in size, suggesting reinnervation of the paretic muscles. The left diaphragm elevation on the chest X-ray also improved. Over a one-year 
A

Right

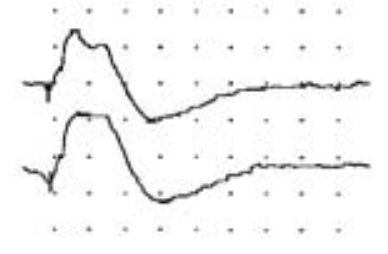

Left

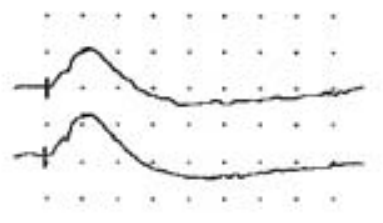

B

\section{Right}
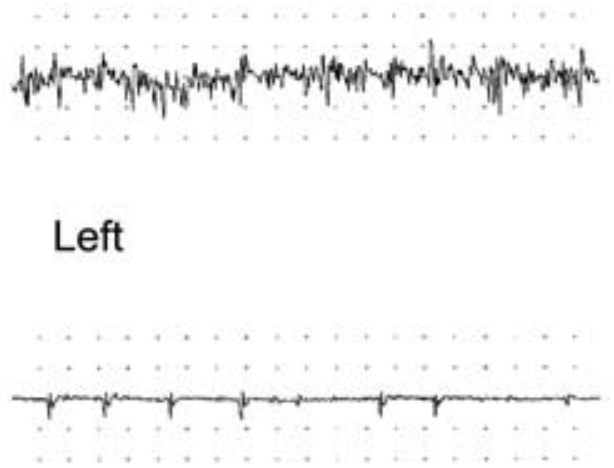

Figure 4. A: $M$ waves of the phrenic nerve electrical stimulation at the neck $(8 \mathrm{~ms} /$ division; 0.2 $\mathrm{mV}$ /division). Two recordings are presented for each nerve. B: Needle EMG findings of the diaphragm during a deep inspiratory phase (20 ms/division; $1 \mathrm{mV} /$ division).

follow-up, the patient showed full recovery of the weakness. Her parkinsonism was also gradually improved after increasing the dosage of carbidopa/levodopa (10/100 $\mathrm{mg}$ four times per day) and cabergoline (4 $\mathrm{mg}$ once a day).

\section{Discussion}

The patient showed acute cervical motor radiculopathy. Her sensory function was preserved, and the electrophysiological studies indicated selective motor involvement. The phrenic nerve was also paretic, indicating the multi-segment spinal motor neurons from C3 to T1 were involved. Her insult was clearly triggered by the akinetic spell, and therefore, infection, intoxication, or adverse effect of the antiparkinson drugs were unlikely to be causes of the palsy.

Judging from the results of the electrophysiological studies, conduction block or conduction slowing at peripheral nerve levels could be ruled out. The denervation potentials of the examined muscles suggest involvement of motor neuron axons or anterior horn cells. On the other hand, the conduction block at the anterior spinal roots is also suggested from the preservation of $\mathrm{M}$ wave amplitude of the median and radial nerves relative to the weakness degree and improvement of $\mathrm{F}$ wave along with the recovery. The markedly reduced interference of the diaphragm motor units on needle EMG in spite of the normal $\mathrm{M}$ wave amplitude also indicates the conduction block of the phrenic nerve, probably at the spinal root site.

The ulnar nerve showed the decreased $M$ wave amplitude at the initial stage in contrast with the median and radial nerves. Considering that the first examination was performed on the 10th day after the weakness onset, this small $\mathrm{M}$ amplitude could not be explained by the Wallerian degeneration after spinal root axonal injury. The patient was frequently akinetic during the down phase of PD, suggesting that the ulnar nerve conduction block at the most distal site (wrist or even more distal) might have been present before the onset. Compression neuropathy at various sites has been reported in PD. Focal compression due to akinesia or body immobilization often induces radial nerve palsy, brachial plexopathy and tarsal tunnel syndrome. The ulnar nerve might be also one of susceptible nerves to local compression.

The most characteristic finding in this case was the normal sensory function, indicating the posterior spinal roots were intact. Differential diagnosis of the radiculopathy limited to the anterior roots was cervical spondylotic amyotrophy and juvenile muscular atrophy of distal upper extremity (Hirayama disease) (6-9). The distinct findings in the present patient from those chronic disorders were the acute onset, multi-segment involvements (C3 to T1), absence of amyotrophy, and fair recovery after the insult. The patient's history of the akinetic spell suggests that the neck was stooped to the left or right side for several hours. This neck position would have induced the root compression at the multiple intervertebral foramina, but this possibility is unlikely since the sensory neurons would be also involved in this case. The other possibility is incomplete or partial spinal root avulsion due to continuous tensile stress on the motor roots. Spinal root avulsion injury dose not usually involve the posterior roots. The anterior spinal roots are more susceptible to stretch or tensile stresses than the posterior roots because of thinner diameters and lower tensile strength, thinner dural sheath, and wider separation of the rootlet at the surface of the spinal cord $(10,11)$. Incomplete root avulsion could be the causes of both axonal injury and conduction block. In addition, the phrenic nerve palsy by stretch injury was also previously reported $(12,13)$.

To be precise, the anterior horn cell involvement could not be fully ruled out in the present patient. Juvenile muscular atrophy of distal upper extremity or flexion cervical myelopathy (Hirayama disease) exhibits amyotrophy of one 
arm, normal sensory function, and anterior horn cell degeneration in autopsy $(8,9)$. This disorder is characteristically caused by forward displacement of the spinal dural sac and compressive flattering of the lower cervical cord during neck flexion (8). The present patient had mild cervical spinal canal stenosis, and neck flexion for several hours might have also involved the anterior horn cells like Hirayama disease. However, after the insult, she did not show any amyotrophy in a chronic stage, and the recovery of muscle weakness was good. This suggests not only sufficient reinnervation of the motor neurons but also recovery of the conduction block. Only flexion myelopathy cannot explain the whole pathomechanism of her disorder.

Cervical spondylosis is a main cause of cervical radiculo- myelopathy, and ischemia of the spinal cord or roots have been assumed as one of its pathmechanisms as well as direct neural compression $(14,15)$. In those cases, the spinal cord often shows intramedullary hyperintensity on MRI, which was absent in the present patient. The wide distribution of the paretic muscles and the selective involvement of motor neurons in our patient suggest the ischemic mechanism to be unlikely.

To our best knowledge, there have been no other reports of acute cervical motor radiculopathy induced by neck or limb immobilization. Clinicians should be more aware of immobilization or akinesia as a cause of acute onset monoplegia particularly in PD patients.

\section{References}

1. Kurlan R, Baker P, Miller C, Shoulson I. Severe compression neuropathy following sudden onset of parkinsonian immobility. Arch Neurol 42: 720, 1985.

2. Preston DN, Grimes JD. Radial compression neuropathy in advanced Parkinson's disease. Arch Neurol 42: 695-696, 1985.

3. Borges LF, Hallett M, Selkoe DJ, Welch K. The anterior tarsal tunnel syndrome. Report of two cases. J Neurosurg 54: 89-92, 1981.

4. Chen R, Collins S, Remtulla H, Parkes A, Bolton CF. Phrenic nerve conduction study in normal subjects. Muscle Nerve 18: 330335, 1995.

5. Bolton CF, Grand'Maison F, Parkes A, Shkrum M. Needle electromyography of the diaphragm. Muscle Nerve 15: 678-681, 1992.

6. Yanagi T, Kato H, Sobue I. Cervical spondylotic amyotrophy simulating motor neuron disease. Rinshou Shinkeigaku (Clin Neurol) 16: 520-528, 1976 (in Japanese).

7. Tsuboi Y, Tokumaru Y, Hirayama K. Clinical differences between "proximal" and "distal" type of cervical spondylotic amyotrophy. Rinshou Shinkeigaku (Clin Neurol) : 147-152, 1995 (in Japanese, Abstract in English).

8. Hirayama K, Tokumaru Y. Cervical dural sac and spinal cord in juvenile muscular atrophy of distal upper extremity. Neurology 54:
1922-1926, 2000.

9. Kuwabara S, Nakajima M, Hattori T, Hirayama K. Electrophysiology of juvenile muscular atrophy of unilateral upper limb (Hirayama's disease). Rinshou Shinkeigaku (Clin Neurol) 39: 508512, 1999 (in Japanese, Abstract in English).

10. Sunderland S, Bradley KC. Stress-strain phenomena in human spinal nerve roots. Brain 84: 120-124, 1961.

11. Sunderland S. Mechanisms of cervical nerve root avulsion in injuries of the neck and shoulder. J Neurosurg 41: 705-714, 1974.

12. Iverson LI, Mittal A, Dugan DJ, Samson PC. Injuries to the phrenic nerve resulting in diaphragmatic paralysis with special reference to stretch trauma. Am J Surg 132: 263-269, 1976.

13. Tiede RH, Hover JR, Davies SF. Unilateral phrenic nerve paralysis from cutting down a Christmas tree. South Med J 87: 11611163, 1994.

14. Shibuya R, Yonenobu K, Yamamoto $K$, et al. Acute arm paresis with cervical spondylosis: three case reports. Surg Neuol 63: 220228, 2005.

15. Mifsud V, Pullicino P. Spinal cord MRI hyperintensities in cervical spondylosis: an ischemic pathogenesis? J Neuroimaging 10: 96-100, 2000.

(C) 2006 The Japanese Society of Internal Medicine http://www.naika.or.jp/imindex.html 